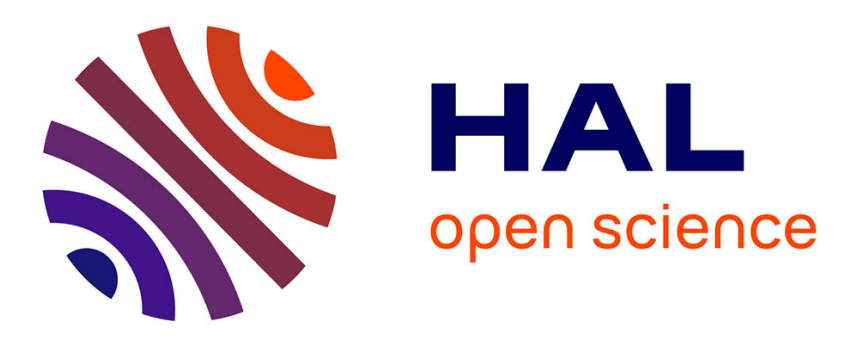

\title{
Impairment of arginine metabolism in rats after massive intestinal resection: Effect of parenteral nutrition supplemented with citrulline versus arginine
}

\author{
Sylwia Osowska, Nathalie Neveux, Samir Nakib, Virginie Lasserre, Luc
}

Cynober, Christophe Moinard

\section{To cite this version:}

Sylwia Osowska, Nathalie Neveux, Samir Nakib, Virginie Lasserre, Luc Cynober, et al.. Impairment of arginine metabolism in rats after massive intestinal resection: Effect of parenteral nutrition supplemented with citrulline versus arginine. Clinical Science, 2008, 115 (5), pp.159-166. 10.1042/CS20070451 . hal-00479420

\section{HAL Id: hal-00479420 https://hal.science/hal-00479420}

Submitted on 30 Apr 2010

HAL is a multi-disciplinary open access archive for the deposit and dissemination of scientific research documents, whether they are published or not. The documents may come from teaching and research institutions in France or abroad, or from public or private research centers.
L'archive ouverte pluridisciplinaire HAL, est destinée au dépôt et à la diffusion de documents scientifiques de niveau recherche, publiés ou non, émanant des établissements d'enseignement et de recherche français ou étrangers, des laboratoires publics ou privés. 


\title{
Impairment of arginine metabolism in rats after massive intestinal resection
}

\section{: Effect of parenteral nutrition supplemented with citrulline versus arginine}

\author{
S. Osowska ${ }^{1}$, N. Neveux ${ }^{1,2}$, S. Nakib ${ }^{2}$, V. Lasserre ${ }^{3}$ L. Cynober ${ }^{1,2}$, C. Moinard ${ }^{1}$
}

${ }^{1}$ Laboratoire de Biologie de la Nutrition, EA 2498, Faculté de Pharmacie - Université Paris Descartes, 4 avenue de l'Observatoire, 75270 Paris Cedex 06, France

${ }^{2}$ Service de Biochimie, Hôtel-Dieu, AP-HP, Paris, France

${ }^{3}$ Biomathématiques et Bioinformatique, Faculté de Pharmacie - Université Paris

Descartes, 4 avenue de l’Observatoire, 75270 Paris Cedex 06, France

\section{Correspondence to: nathalie.neveux@nutrition-paris5.fr}

Laboratoire de Biologie de la Nutrition (case 16), Faculté de Pharmacie Université Paris Descartes, 4 avenue de l'Observatoire, 75270 Paris Cedex 06

Tél. 33 (1) 53739945 - Fax. 33 (1) 53739952

Key words: malnutrition, citrulline, arginine, short bowel syndrome, intestinal adaptation.

Acknowledgements: S. Osowska was the recipient of a fellowship from Laboratoires Biocodex. This research was partly supported by a grant from Laboratoires Biocodex and partly by a grant from the French Ministry of Research and Technology (4-year contract EA 2498). 


\section{Abstract}

Background: Arginine homeostasis is impaired in short bowel syndrome, but its supplementation in short bowel syndrome patients remains controversial. Recently, we demonstrated the benefits of citrulline supplementation by enteral route in resected rats. Since the first step in managing short bowel syndrome is to initiate total parenteral nutrition, we hypothesized that parenteral citrulline supplementation would be more appropriate in this situation than arginine supplementation. Material and methods: 24 rats were assigned to 4 groups. The Sham group underwent transection while the 3 other groups underwent resection (R) of $80 \%$ of the small intestine. All rats were then fed exclusively by total parenteral nutrition as follows: supplementation with citrulline $(\mathrm{R}+\mathrm{CIT})$, with arginine $(\mathrm{R}+\mathrm{ARG})$, or no supplementation (R). All the rats received isocaloric and isonitrogenous nutrition for 4 days. Nitrogen balance was measured daily. At sacrifice, the blood was collected and the intestinal mucosa and Extensor Digitorum Longus muscle were removed for amino acid and protein analysis. Results: Citrulline and arginine increased mucosal protein content in the ileum (vs. Sham and $\mathrm{R}, \mathrm{p}<0.05)$. However, only citrulline prevented Extensor Digitorum Longus atrophy (R+CIT: $130 \pm 3$ vs. R: $100 \pm 6$ and R+ARG: $110 \pm 2 \mathrm{mg}, \mathrm{p}<0.05$ ). In addition, arginine worsened nitrogen balance (R+ARG: $104 \pm 46$ vs. R: 249 \pm 69 mg/72 hrs, $\mathrm{p}<0.05$ ). Conclusion: only citrulline was able to prevent muscle atrophy and it was achieved independently from any noticeable effect on the gut in particular because citrulline and arginine share the same effect on mucosal ileal protein content. These data suggest that citrulline should be considered as a potential supplement for total parenteral nutrition of short bowel syndrome patients. 


\section{Introduction}

Arginine (ARG) presents numerous metabolic functions as a precursor of nitric oxide, agmatine, proline, polyamines and creatine [1]. Furthermore, ARG stimulates hormone secretion (i.e. insulin and growth hormone) [2]. Finally, it plays a key role in nitrogen homeostasis through the control of ureagenesis [3].

ARG homeostasis results from tight cooperation between the intestine and the kidneys, and citrulline (CIT) has a central role in this homeostatic process. At whole-body level, the intestine is the main site of CIT production because it is the only tissue that contains all the enzymes required to synthesize CIT from either ARG or glutamine (GLN; see schema) [4]. The kidneys (which express argininosuccinate synthase and argininosuccinate lyase) extract about $75 \%$ of plasma CIT, which represents $83 \%$ of the CIT released by the gut [5-8].

These metabolic considerations explain why short bowel syndrome (SBS) is characterized by profound perturbations of ARG metabolism [5] and why some authors [9;10] have suggested that ARG is in fact an essential amino acid in SBS. However, we recently demonstrated [11] that CIT supplementation is more efficient than ARG in increasing ARG pools and restoring nitrogen balance after massive intestinal resection in rats. In our previous work, CIT was administered by enteral route. Since the liver and the intestine play a major role in ARG metabolism, it may be hypothesized that the route of administration (i.e. parenteral route, which bypasses the splanchnic area, versus the enteral one) may profoundly affect the metabolism of the administered CIT and ARG. The effect would probably be more pronounced on ARG than CIT, which is neither metabolized in the gut nor taken up or released by the liver [1;3]. The aim of this work was to evaluate the effects of CIT and ARG administered by parenteral route in a rat model of massive intestinal resection. 


\section{Materials and Methods}

Chemicals: All chemicals were purchased from Sigma (St-Quentin-Fallavier, France). CIT was a gift from Biocodex (Compiègne, France).

Animals: Male Wistar rats ( $n=24$, body weight 220-230 g) purchased from Charles River (L’Arbresle, France) were housed in metabolic cages for a 5-day acclimatization period before surgery, and were given free access to standard laboratory chow (17\% protein, 3\% fat, 59\% carbohydrate, 21\% water, vitamins and minerals) (A04, Usine d'Alimentation Rationnelle, Villemoisson-sur-Orge, France) and water.

Surgical procedures: The animals were fasted for $12 \mathrm{~h}$ before surgery. They were anesthetized by isoflurane inhalation (3\% isoflurane) using a regulated airflow apparatus (Minerve, Esternay, France). Enterectomy was performed on the rats by removing $80 \%$ of the small intestine, leaving $10 \%$ of the proximal jejunum and $10 \%$ of the distal ileum, as previously described [11]. Six rats were sham-operated (transection). For both resected and transected animals, continuity was restored with end-to-end anastomosis using a single running silk 6-0 suture. Immediately after intestinal resection or transection, the catheter was fitted into the jugular vein. A silicone tube (Tygon, size $0.51 \mathrm{~mm}$, Fisher Bioblock Scientific, Illkirch, France) was introduced into the vein and the remaining end of the catheter was tunneled subcutaneously to the back of the neck and attached to a spring coil-swivel mechanism (Harvard Apparatus, Les Ulis, France) allowing the rats to move freely within the cage [12;13]. Before the surgical procedure, all the rats received a painkiller (Temgesic ${ }^{\circledR}$, Schering-Plough, Levallois-Perret, France) by the subcutaneous route at a dose of $0.05 \mathrm{mg} / \mathrm{kg}$ body weight.

One of the authors (C.M.) is authorized by the French government (No. 75522) to use this experimental model of surgery. Animal care complied with French regulations for the 
protection of animals used for experimental and other scientific purposes (D 2001-486) and with European Community regulations (Official Journal of the European Community L538 12:18:1986).

Postoperative care: The rats were housed individually in metabolic cages. Parenteral nutrition was started immediately after surgery. Parenteral nutrition was given using a Harvard infusion pump (Pump 11, Harvard Apparatus, Les Ulis, France). During the first 24 hours of parenteral nutrition, the flow rate was increased progressively to reach a final rate of $300 \mathrm{ml} / \mathrm{kg}$ body weight/day, which corresponds to a nitrogen intake of $2 \mathrm{~g} / \mathrm{kg} / \mathrm{day}$ and $260 \mathrm{kcal} / \mathrm{kg} /$ day energy, covering nutritional requirements in rats (11-13). The rats had free access to water.

Experimental groups: The rats were randomly assigned to one of four groups:

- In the citrulline group (R+CIT, $n=6)$, the rats received standard parenteral nutrition (Vintène $^{\circledR}$, Baxter, Maurepas, France) (Table 1) supplemented with CIT at a dose of 1 g/kg/day (5.71 mmol/kg/day).

- In the arginine group $(\mathrm{R}+\mathrm{ARG}, n=6)$, the rats received the standard parenteral nutrition supplemented with ARG at a dose of $0.994 \mathrm{~g} / \mathrm{kg} /$ day, making it equimolar to the CIT group.

- In the control group $(R, n=6)$ and the sham group (Sham, $n=6)$, the rats received standard parenteral nutrition and were either resected (R) or transected (Sham).

In all groups, energy was supplied as carbohydrate (glucose $50 \%$ ) and long-chain triglycerides containing fat emulsion (Endolipid $20 \%$, B. Braun Medical, Boulogne, France). The nutritional regimen also provided electrolytes (Standard III, Aguettant, Lyon, France) and water. All the groups received isonitrogenous $\left(2 \mathrm{~g} \mathrm{~N} / \mathrm{kg} /\right.$ day, adjusted adding Vintene ${ }^{\circledR}$ in $\mathrm{R}$, sham and CIT groups) and isocaloric (260 kcal/kg/day) nutrition. Nitrogen and calorie intakes 
and amounts of CIT or ARG mimicked those used in clinical nutrition, which takes into account the fact that metabolic rate is ten-fold higher in rats than in humans [11;14;15].

The rats were weighed and urine was collected daily. Total parenteral nutrition was administered for 4 days. Parenteral nutrition was stopped $2 \mathrm{~h}$ before sacrifice by decapitation.

\section{Tissue removal}

Blood was sampled into heparinized tubes, which were rapidly centrifuged.

Jejunum and ileum: The intestine was resected from the ligament of Treitz to the ileocecal junction. The mucosa was washed with $\mathrm{NaCl}(0.9 \%)$, everted and scraped off, then weighed, rapidly frozen in liquid nitrogen and stored at $-80^{\circ} \mathrm{C}$ until analysis.

Muscles: The extensor digitorum longus (EDL) was rapidly removed, weighed, then frozen in liquid nitrogen and stored at $-80^{\circ} \mathrm{C}$ until analysis.

\section{Parameters under study and analytical methods}

Amino acid (AA) concentrations in plasma and tissues: Plasma was deproteinized with a 30\% $(w / v)$ sulfosalicylic acid solution and the supernatants were stored at $-80^{\circ} \mathrm{C}$ until analysis. Tissues were ground and deproteinized with $10 \%$ trichloroacetic acid (TCA) containing $0.5 \mathrm{mM}$ EDTA, and the supernatants were stored at $-80^{\circ} \mathrm{C}$ until analysis. AAs were measured by ion exchange chromatography [16] using an amino acid analyzer (AminoTac, JLC-500/V, Jeol, Tokyo, Japan). The results of our participation in the European Quality Control Scheme (ERNDIM, Maastricht, The Netherlands) indicate the accuracy of our AA determinations.

Polyamines: Dansyl derivatives of intestinal polyamines were assayed according to the method of Seiler and Knodgen [17], with some modifications. Intestine samples were homogenized in a phosphate-buffer $\left(50 \mathrm{mM} \quad \mathrm{KH}_{2} \mathrm{PO}_{4} / \mathrm{Na}_{2} \mathrm{HPO}_{4}, 300 \mu \mathrm{l}\right.$ of buffer per mg of tissue). Homogenates were centrifuged for $5 \mathrm{~min}$ at $3000 \mathrm{~g}$. Supernatants were diluted 15 times in 
phosphate buffer and stored at $-80^{\circ} \mathrm{C}$ until analysis. Samples were incubated for 2 hours at $60^{\circ} \mathrm{C}$ in the dark with $0.5 \mathrm{ml}$ of dansyl chloride $(5 \mathrm{mg} / \mathrm{ml}$ in acetone). The solution was then evaporated under nitrogen and extraction was performed using $8 \mathrm{ml}$ of $30 \%$ methanol (v/v). The organic phase was collected and evaporated, and the dansyl derivatives were resuspended in $0.1 \mathrm{ml}$ of the initial mobile phase and 20 microliters were injected into the chromatograph. Dansylputrescine, dansylspermidine and dansylspermine were separated on a C8 column (length: $15 \mathrm{~cm}$, particle diameter: $5 \mu \mathrm{m}$ ) using the 5500 Vista Varian pump system (Varian, Palo Alto, USA). After fluorimetric detection, polyamine concentrations were estimated by the internal standard method (standard: hexanediamine). Results are expressed as nmol per gram of tissue.

Nitrogen was quantified by chemiluminescence [18] using an Antek 9000 apparatus (Antek, Houston, TX), and nitrogen balance was calculated as the difference between nitrogen intake and urinary nitrogen output.

\section{Statistical analysis}

Data are expressed as means \pm SEM. Comparisons between sets of data were made using oneway analysis of variance (ANOVA) followed by the Duncan test. PCSM software was used (Deltasoft, Grenoble, France). Differences at $p<0.05$ were considered significant. 


\section{Results}

Body weight loss (Figure 1) (i.e. the difference between body weight at the beginning of TPN administration and the end of the experiment) did not differ significantly in the four studied groups.

Tissue weight (Table 2). Gut resection led to muscle atrophy ( $R$ vs. Sham, $p<0.05$ ). However, EDL weight was restored by CIT supplementation $(R+C I T$ vs. $R$ and $R+A R G, \mathrm{p}<0.05)$. ARG and CIT supplementation increased mucosal ileum weight $(R+C I T$ and $R+A R G$ vs. Sham, $\mathrm{p}<0.05)$ whereas only the CIT-treated group had higher mucosal jejunum weight than sham (vs. Sham, $\mathrm{p}<0.05)$.

Tissue protein contents (Table 3). There were no significant changes in protein content in EDL muscle. Intestinal resection led to a significant increase in mucosal protein content in the jejunum ( $R$ vs. sham, $\mathrm{p}<0.05)$ without a significant additional effect of ARG or CIT supplementation. The mucosal protein content of the ileum, which was not modified by resection, was significantly increased in both the ARG- and CIT-supplemented groups $(R+A R G$ and $R+C I T$ vs. Sham and $R, \mathrm{p}<0.05)$.

Amino acid concentrations. CIT was the only amino acid whose profile was influenced by resection or by the different diets (Table 4A).

- Plasma. Intestinal resection led to a decrease in CIT levels compared with the sham group ( $R$ vs. Sham, $p<0.05$ ). CIT administration led to an increase in plasma CIT concentrations ( $R+C I T$ vs. Sham, $R, R+A R G, p<0.05$ ). In the CIT group, the increase in EDL weight was positively correlated with plasma CIT concentration $(r=0.73, p<0.0001)$. 
- Muscles. Intestinal resection led to a decrease in muscle CIT levels ( $R$ vs. Sham, $\mathrm{p}<0.05)$. Only CIT supplementation led to a significant increase in muscle CIT levels $(R+C I T$ vs. Sham, $R, R+\mathrm{ARG}, \mathrm{p}<0.05)$.

- Intestinal mucosa. After intestinal resection, there was a decrease in CIT content in the mucosal jejunum ( $R$ vs. Sham, $\mathrm{p}<0.05$ ). While both CIT and ARG led to an increased CIT content in the mucosal jejunum, only CIT supplementation was able to completely restore mucosal jejunum CIT content $(R+C I T$ vs. $R$ and $R+A R G, \mathrm{p}<0.05)$.

There were no treatment effects on ARG levels (Table 4B) nor on other AAs (data not shown).

Polyamine contents in intestinal mucosa (Table 5). Intestinal resection was associated with a significant increase in spermidine $(R$ vs. Sham, $\mathrm{p}<0.05)$. This higher level of spermidine content was also found in the mucosal jejunum from the CIT-supplemented group $(R+C I T v s$. Sham: $\mathrm{p}<0.05)$ but not in the ARG-supplemented group $(R+A R G$ vs. Sham: ns). There was no difference in spermine or putrescine contents between the groups studied.

Nitrogen balance (Figure 2). Cumulative nitrogen balance was significantly lower in the three resected groups compared with the Sham group (Sham vs. $R, R+A R G$ and $R+C I T, \mathrm{p}<0.05$ ), especially when the diet was supplemented with ARG $(\mathrm{R}+A R G$ vs. $R, \mathrm{p}<0.05)$. 


\section{DISCUSSION}

The intestine is an important organ for the maintenance of ARG homeostasis and therefore for whole-body nitrogen homeostasis. The pioneering study by Crenn et al [19] together with earlier experimental data [9;10] suggest that ARG administration could become critical in SBS. In fact, the metabolic specificities of ARG and its related AAs (see introduction) together with our recent research [11] show that it is CIT administration rather than ARG which could be a better strategy in an SBS setting.

As expected, there was a two-fold increase in plasma CIT concentrations after parenteral CIT administration. This result is in agreement with our previous work using enteral infusion [11]. However, CIT supplementation did not affect ARG concentration in either the plasma or in the tissues. This is a surprising result since we previously observed that the same amount of CIT (i.e. $1 \mathrm{~g} / \mathrm{kg} / \mathrm{d}$ ) given by enteral route is able to generate a large amount of ARG in resected animals [11]. The main factor determining ARG synthesis by the kidney is plasma CIT concentration [6]. Since renal function, assessed on the basis of urinary creatinine, was not altered in our model (data not shown), it is likely that ARG synthesis was not compromised. Data from the literature $[5 ; 7 ; 8]$ indicate that ARG catabolism is the main regulator of ARG homeostasis. Using a similar model of enterectomy and labelled isotopes, Dejong et al [20] demonstrated, that net renal CIT uptake and ARG release were both decreased by $50 \%$ due to intestinal resection. However, despite decreased net renal CIT consumption and renal ARG production in enterectomized rats, neither the whole-body rate of ARG appearance nor arterial ARG were modified, prompting the same group [21] to conclude that intestinal resection does not have important effects on whole-body ARG production. Therefore, our data clearly show that ARG administration failed to increase ARG pools. The dosage used cannot be incriminated since the same dose $(0.994 \mathrm{~g} / \mathrm{kg} / \mathrm{day})$ given by enteral route in the same SBS rat model was associated with a large increase in plasma and muscle arginine content (11). This suggests that ARG is intensively metabolized in response to intestinal resection and that ARG 
metabolism is clearly affected by route of administration (Sham + standard enteral nutrition: ARG: $110 \pm 12 \mu \mathrm{mol} / \mathrm{L}$ (from [11]) and Sham + standard parenteral nutrition: ARG: $246 \pm 24$ $\mu \mathrm{mol} / \mathrm{L})$. This may reflects the importance of splanchnic first-pass in ARG metabolism. However, the time schedule of the present study was different from our earlier one [11] and so comparison of data requires caution. The few data available on parenteral supplementation in SBS rats [21;22] actually did not use the same route of administration (i.e. ARG was administered subcutaneously, which does not reflect an intravenous administration). Another explanation is that the rats were studied earlier than in our previous study [11] making the rats more catabolic with higher ARG turnover. Finally, in certain pathological situations, the liver appears to be able to extract CIT [23;24] by diverting this amino acid from kidney capture. The results we obtained using the enteral route [11] and the parenteral route, show that CIT reaching the liver by the arterial artery has a different behavior from CIT coming from the portal vein. This point deserves further studies. Furthermore, studies using labeled amino acids and arterio-venous difference measurements are required to define ARG use at whole-body and organ levels, following resection.

Our results suggest that ARG and CIT increase mucosal weight and mucosal protein content. We also observed that these effects could differ according to the intestinal section (jejunum or ileum). This difference may be related to differences in the adaptation process that are not yet understood. These data are promising results which suggest that CIT and ARG could affect gut protein content. It would be interesting to extend our investigation to specific markers of intestinal adaptation (i.e. bowel circumference, bowel weight, mucosa DNA content, villus height and crypt depth) and to determine the mechanism involved in this action. The effect of ARG on intestinal adaptation remains controversial $[9 ; 10 ; 20 ; 22 ; 25 ; 26]$. At the molecular level, the synthesis of intracellular polyamines is a critical event for the onset of mucosal hyperplasia. Hence, these molecules play an important role in the maintenance of intestinal mucosa [27]. This is well illustrated in SBS, since a significant increase in the 
polyamine content of the remaining intestine was reported in the literature [20;28]. However, in our study, only spermidine was increased in the jejunum ( $\mathrm{R}$ and $\mathrm{R}+\mathrm{CIT}$ vs Sham, $\mathrm{p}<0.05)$. This discrepancy with the literature may be because polyamine content usually increases in the first hours following resection (less than 24 hours) [29;30]. In the present study, polyamine assays were performed 96 hours after resection, and the absence of the significantly increased polyamine content after this period is consistent with the studies cited above.

Alternatively, ARG, when given parenterally, may be used preferentially by other tissues. As expected, resected animals exhibited a decreased nitrogen balance. While CIT had no effect on nitrogen balance, ARG clearly appeared to have a deleterious effect. This could be explained as follows: as arginase activity is very high in the liver [31], most of the ARG that reaches the liver is converted into urea. This fact was well illustrated by de Bandt et al.'s study [32] in a model of isolated perfused liver. These authors demonstrated that ARG was extensively taken up by the liver and rapidly released as ORN and urea, which supports ARGinduced acceleration of amino acid catabolism and activation of the urea cycle. However, CIT, which is not taken up by the liver [33], limits the activation of ureagenesis, making it a candidate therapy for preserving nitrogen balance. However, this study using parenteral administration did not reproduce the beneficial effect of CIT on nitrogen balance observed when CIT is administered by enteral nutrition [11]. As for the ARG levels, this discrepancy may be explained by the difference in the duration of the treatment ( 3 days in this work vs. 10 days previously), because the beneficial effect of CIT was observed only after the $4^{\text {th }}$ day of treatment.

Finally, EDL weight was increased in the CIT-supplemented group compared to both the control and ARG-supplemented groups. Interestingly, this increase was positively correlated with plasma CIT concentrations $(\mathrm{r}=0.73, \mathrm{p}<0.0001)$. This unexpected funding could be related to the ability of CIT to stimulate muscle protein synthesis [34]. We recently showed [35] that CIT administration to malnourished old rats stimulates muscle protein 
synthesis, which could explain the trend in increased muscle protein content observed in the present study. In addition, our recent research [36], using isolated incubating muscles suggests that CIT is able to directly affect protein synthesis. However, to the best of our knowledge, our work is pioneering and there is no data available in the literature that might help us to explain this effect. Alternatively, a modification of water content might be involved. Unfortunately, this variable was not measured in this study. However, we previously measured muscle water content in a number of studies in several physiopathological conditions (aging, enteral or parenteral nutrition, stress, malnutrition, SBS, etc.) and it was never modified by treatment. The present work does not enable us to draw definitive conclusion: further work to assess body composition (using DEXA or $\mathrm{D}_{2} \mathrm{O}$ methods), whole body and tissues protein synthesis would help to determine the precise influence of CIT on lean body mass in various tissues and organs. In conclusion, parenteral CIT supplementation restores the plasma CIT pools that are reduced after intestinal resection. However, our results are different from those obtained after enteral administration. This suggests that the route of administration (i.e. enteral vs. parenteral) has profoundly different effects on ARG metabolism. CIT shares with ARG the positive effect on intestinal adaptation in terms of intestinal mucosa weight and protein content. However, only CIT also has a positive effect on muscle weight and importantly, ARG administration worsens nitrogen balance, whereas CIT does not. These data suggest that CIT could be potentially considered as a supplement in parenteral nutrition for the patients with SBS in order to preserve nutritional state and promote intestinal adaptation. Together with our previous work [11], these results open the way to clinical trials in patients suffering from SBS. 


\section{Reference List}

1. Wu, G and Morris Jr,S.M. (2004) Arginine in mammals. In Metabolic and therapeutic aspects of amino acids in clinical nutrition (Cynober,L., ed.), CRC Press, Boca Raton

2. Collier,S.R., Casey,D.P. and Kanaley,J.A. (2005) Growth hormone responses to varying doses of oral arginine. Growth Horm.IGF.Res. 15, 136-139.

3. Cynober,L., Le Boucher,J. and Vasson,M.P. (1995) Arginine metabolism in mammals. J.Nutr.Biochem. 6, 402-413.

4. Curis,E., Nicolis,I., Moinard,C. et al. (2005) Almost all about citrulline in mammals. Amino Acids 29, 177-205.

5. Wakabayashi,Y. (2004) The glutamate crossway. In Metabolic and therapeutic aspects of amino acids in clinical nutrition (Cynober,L., ed.), CRC Press, Boca Raton

6. Dhanakoti,S.N., Brosnan,J.T., Herzberg,G.R. and Brosnan,M.E. (1990) Renal arginine synthesis: studies in vitro and in vivo. Am.J Physiol 259, E437-E442.

7. Castillo,L., Chapman,T.E., Sanchez,M. et al. (1993) Plasma arginine and citrulline kinetics in adults given adequate and arginine-free diets. Proc.Natl.Acad.Sci.U.S.A 90, 7749-7753.

8. Castillo,L., Sanchez,M., Vogt,J. et al. (1995) Plasma arginine, citrulline, and ornithine kinetics in adults, with observations on nitric oxide synthesis. Am.J Physiol 268, E360E367.

9. Wakabayashi,Y., Yamada,E., Yoshida,T. and Takahashi,H. (1994) Arginine becomes an essential amino acid after massive resection of rat small intestine. J.Biol.Chem. 269, 32667-32671.

10. Wakabayashi,Y., Yamada,E., Yoshida,T. and Takahashi,N. (1995) Effect of intestinal resection and arginine-free diet on rat physiology. Am.J.Physiol 269, G313-G318.

11. Osowska S., Moinard,C., Neveux,N., Loï C and Cynober,L. (2004) Citrulline increases arginine pools and restores nitrogen balance after massive intestinal resection. Gut 53, 1781-1786.

12. Osowska,S., De Bandt,J.P., Chaib,S., Neveux,N., Berard,M.P. and Cynober,L. (2003) Efficiency of a cysteine-taurine-threonine-serine supplemented parenteral nutrition in an experimental model of acute inflammation. Intensive Care Med. 29, 1798-1801.

13. Loi,C., Kana,G., Blanc,M.C., Genthon,C. and Cynober,L. (2005) Evaluation of a glycine-rich amino acid solution for parenteral nutrition in endotoxemic rats. Crit Care Med. 33, 2344-2349.

14. Moinard,C., Chauveau,B., Walrand,S. et al. (1999) Phagocyte functions in stressed rats: modulation by glutamine, arginine and ornithine 2-oxoglutarate compared. Clin.Sci. 97, 59-65. 
15. Loi,C., Osowska,S., Neveux,N., Darquy,S., Cynober,L. and Moinard,C. (2005) Effects of an immune-enhancing diet in endotoxemic rats. Nutrition 21, 255-263.

16. Neveux,N., David,P. and Cynober,L. (2004) Measurement of amino acid concentration in biological fluids and tissues using ion-exchange chromatography. In Metabolic and therapeutic aspects of amino acids in clinical nutrition (Cynober,L., ed.), CRC Press, Boca Raton

17. Seiler,N. and Knodgen,B. (1980) High-performance liquid chromatographic procedure for the simultaneous determination of the natural polyamines and their monoacetyl derivatives. J Chromatogr. 221, 227-235.

18. Grimble,G. (1991) Measurement of elemental nitrogen by chemiluminescence [letter; comment]. JPEN J.Parenter.Enteral Nutr. 15, 696.

19. Crenn,P., Coudray-Lucas,C., Thuillier,F., Cynober,L. and Messing,B. (2000) Postabsorptive plasma citrulline concentration is a marker of absorptive enterocyte mass and intestinal failure in humans. Gastroenterology 119, 1496-1505.

20. Dejong,C.H., Welters,C.F., Deutz,N.E., Heineman,E. and Soeters,P.B. (1998) Renal arginine metabolism in fasted rats with subacute short bowel syndrome.

Clin.Sci.(Colch.) 95, 409-418.

21. Welters,C.F., Dejong,C.H., Deutz,N.E. and Heineman,E. (1999) Effects of parenteral arginine supplementation on the intestinal adaptive response after massive small bowel resection in the rat. J.Surg.Res. 85, 259-266.

22. Sukhotnik,I., Mogilner,J.G., Lerner,A. et al. (2005) Parenteral arginine impairs intestinal adaptation following massive small bowel resection in a rat model. Pediatr.Surg.Int. 21, 460-465.

23. van de Poll,M.C., Siroen,M.P., van Leeuwen,P.A. et al. (2007) Interorgan amino acid exchange in humans: consequences for arginine and citrulline metabolism. Am.J Clin Nutr. 85, 167-172.

24. van de Poll,M.C., Ligthart-Melis,G.C., Boelens,P.G., Deutz,N.E., van Leeuwen,P.A. and Dejong,C.H. (2007) Intestinal and hepatic metabolism of glutamine and citrulline in humans. J Physiol 581, 819-827.

25. Sukhotnik,I., Lerner,A., Sabo,E. et al. (2003) Effects of enteral arginine supplementation on the structural intestinal adaptation in a rat model of short bowel syndrome. Digestive Diseases and Sciences 48, 1346-1351.

26. Ozturk,H., Dokucu,A.I., Yagmur,Y. and Sari,I. (2002) Effects of supplemental Larginine on the intestinal adaptive response after massive small-bowel resection in rats. Pediatric Surgery International 18, 332-336.

27. Moinard,C., Cynober,L. and De Bandt,J.P. (2005) Polyamines: metabolism and implications in human diseases. Clin Nutr. 24, 184-197.

28. Dumas,F., De Bandt,J.P., Colomb,V. et al. (1998) Enteral ornithine alpha-ketoglutarate enhances intestinal adaptation to massive resection in rats. Metabolism 47, 1366-1371. 
29. Rountree,D.B., Ulshen,M.H., Selub,S. et al. (1992) Nutrient-independent increases in proglucagon and ornithine decarboxylase messenger RNAs after jejunoileal resection. Gastroenterology 103, 462-468.

30. Segovia-Silvestre,T., Pita,A.M., Vilar,L., Venereo,Y., Orta,X. and Farriol,M. (2001) Intestinal ornithine decarboxylase in short bowel syndrome patients with oral diet. Clin Nutr. 20, 171-175.

31. Morris,S.M., Jr. (2006) Arginine: beyond protein. Am.J Clin Nutr. 83, 508S-512S.

32. De Bandt,J.P., Cynober,L., Lim,S.K., Coudray-Lucas,C., Poupon,R. and Giboudeau,J. (1995) Metabolism of ornithine, alpha-ketoglutarate and arginine in isolated perfused rat liver. Br.J.Nutr. 73, 227-239.

33. Drotman,R.B. and Freedland,R.A. (1972) Citrulline metabolism in the perfused rat liver. Am.J Physiol 222, 973-975.

34. Moinard,C. and Cynober,L. (2007) Citrulline: a new player in the control of nitrogen homeostasis. J.Nutr. 137, 1621S-1625S.

35. Osowska,S., Duchemann,T., Walrand,S. et al. (2006) Citrulline modulates muscle protein metabolism in old malnourished rats. Am.J Physiol Endocrinol.Metab 291, E582-E586.

36. Moinard,C., Jourdan M, Walrand,S., Le Plenier,S., Boirie,Y. and Cynober,L. (2007) Citrulline directly modulates muscle protein synthesis: evidence from in vitro studies. Clin.Nutr. 26 (suppl.2), 155-156 (Abstract). 
Table 1. Composition of the amino acid solution (mmol/l)

\begin{tabular}{lc}
\hline Essential amino acids & \\
\hline L-Isoleucine & 53 \\
L-Leucine & 107 \\
L-Valine & 60 \\
L-Lysine & 68 \\
L-Phenylalanine & 55 \\
L-Methionine & 47 \\
L-Threonine & 46 \\
L-Tryptophan & 12 \\
\hline Non-essential amino acids & \\
\hline L-Aspartate & 23 \\
L-Serine & 29 \\
L-Glutamate & 34 \\
L-Proline & 96 \\
Glycine & 123 \\
L-Alanine & 146 \\
L-Histidine & 26 \\
L-Arginine & 86 \\
L-Tyrosine & 2 \\
Acetylcysteine & 13 \\
\hline & \\
\hline & \\
\hline & \\
\hline
\end{tabular}


Table 2. Muscle and intestine mucosa weight.

\begin{tabular}{ccccc}
\hline \hline & Sham & R & R+ARG & R+CIT \\
\hline EDL (mg) & $120 \pm 6^{\mathrm{a}, \mathrm{c}}$ & $100 \pm 6^{\mathrm{b}}$ & $110 \pm 2^{\mathrm{a}, \mathrm{b}}$ & $130 \pm 3^{\mathrm{c}}$ \\
Jejunum (mg/cm) & $36 \pm 6^{\mathrm{a}}$ & $48 \pm 6^{\mathrm{a}, \mathrm{b}}$ & $52 \pm 3^{\mathrm{a}, \mathrm{b}}$ & $65 \pm 9^{\mathrm{b}}$ \\
Ileum (mg/cm) & $26 \pm 2^{\mathrm{a}}$ & $44 \pm 5^{\mathrm{a}, \mathrm{b}}$ & $54 \pm 3^{\mathrm{b}}$ & $52 \pm 9^{\mathrm{b}}$ \\
\hline \hline
\end{tabular}

Four groups of rats were studied: Sham (transected rats fed standard parenteral nutrition), $R$ (resected rats fed standard parenteral nutrition), $R+A R G$ (resected rats fed an argininesupplemented formula), $R+C I T$ (resected rats fed a citrulline-supplemented formula) Results are expressed as means \pm SEM.

ANOVA + Duncan's test. Values in a row with different superscript letters are statistically different at $\mathrm{p}<0.05$.

Table 3. Protein content in the intestinal mucosa and EDL muscle.

\begin{tabular}{ccccc}
\hline \hline & Sham & R & R+ARG & R+CIT \\
\hline EDL (mg) & $11.9 \pm 1.9$ & $10.1 \pm 1.4$ & $11.9 \pm 0.9$ & $13.6 \pm 0.8$ \\
Jejunum (mg/g) & $46 \pm 3^{\mathrm{a}}$ & $57 \pm 7^{\mathrm{b}}$ & $72 \pm 4^{\mathrm{b}}$ & $73 \pm 6^{\mathrm{b}}$ \\
Ileum (mg/g) & $51 \pm 3^{\mathrm{a}}$ & $53 \pm 7^{\mathrm{a}}$ & $71 \pm 5^{\mathrm{b}}$ & $72 \pm 3^{\mathrm{b}}$ \\
\hline \hline
\end{tabular}

Four groups of rats were studied: Sham (transected rats fed standard parenteral nutrition), $R$ (resected rats fed standard parenteral nutrition), $R+A R G$ (resected rats fed an argininesupplemented formula), $R+C I T$ (resected rats fed a citrulline-supplemented formula) Results are expressed as means \pm SEM.

ANOVA + Duncan's test. Values in a row with different superscript letters are statistically different at $\mathrm{p}<0.05$. 
Table 4. Citrulline (A) and arginine (B) concentrations in plasma and tissues.

\begin{tabular}{ccccc}
\hline \hline A- Citrulline & Sham & R & R+ARG & R+CIT \\
\hline Plasma $(\mu \mathrm{mol} / \mathrm{L})$ & $80 \pm 6^{\mathrm{a}}$ & $46 \pm 2^{\mathrm{b}}$ & $41 \pm 5^{\mathrm{c}}$ & $114 \pm 7^{\mathrm{d}}$ \\
EDL $(\mathrm{nmol} / \mathrm{g})$ & $167 \pm 16^{\mathrm{a}}$ & $79 \pm 7^{\mathrm{b}}$ & $100 \pm 16^{\mathrm{c}}$ & $502 \pm 27^{\mathrm{d}}$ \\
Jejunum $(\mathrm{nmol} / \mathrm{g})$ & $145 \pm 25^{\mathrm{a}}$ & $59 \pm 10^{\mathrm{b}}$ & $63 \pm 17^{\mathrm{c}}$ & $143 \pm 33^{\mathrm{a}}$ \\
Ileum $(\mathrm{nmol} / \mathrm{g})$ & $141 \pm 35$ & $53 \pm 13$ & $86 \pm 21$ & $156 \pm 37$ \\
\hline \hline
\end{tabular}

\begin{tabular}{ccccc}
\hline \hline B- Arginine & & & & \\
\hline Plasma $(\mu \mathrm{mol} / \mathrm{L})$ & $246 \pm 24$ & $228 \pm 31$ & $244 \pm 25$ & $288 \pm 19$ \\
EDL $(\mathrm{nmol} / \mathrm{g})$ & $1200 \pm 163$ & $1071 \pm 204$ & $951 \pm 122$ & $1131 \pm 184$ \\
Jejunum $(\mathrm{nmol} / \mathrm{g})$ & $304 \pm 63$ & $444 \pm 80$ & $467 \pm 66$ & $200 \pm 61$ \\
Ileum $(\mathrm{nmol} / \mathrm{g})$ & $200 \pm 58$ & $401 \pm 113$ & $256 \pm 62$ & $190 \pm 31$ \\
\hline \hline
\end{tabular}

Four groups of rats were studied: Sham (transected rats fed standard parenteral nutrition), $R$ (resected rats fed standard parenteral nutrition), $R+A R G$ (resected rats fed an argininesupplemented formula), $R+C I T$ (resected rats fed a citrulline-supplemented formula) Results are expressed as means \pm SEM.

ANOVA + Duncan's test. Values in a row with superscript letters are statistically different at $\mathrm{p}<0.05$.

Table 5. Polyamine content in the intestinal mucosa.

\begin{tabular}{ccccc}
\hline \hline Jejunum & Sham & $\mathrm{R}$ & $\mathrm{R}+\mathrm{ARG}$ & $\mathrm{R}+\mathrm{CIT}$ \\
\hline putrescine & $55 \pm 14$ & $89 \pm 11$ & $70 \pm 12$ & $84 \pm 14$ \\
spermidine & $697 \pm 62^{\mathrm{a}}$ & $1004 \pm 46^{\mathrm{b}, \mathrm{c}}$ & $821 \pm 57^{\mathrm{a}, \mathrm{c}}$ & $987 \pm 100^{\mathrm{b}, \mathrm{c}}$ \\
spermine & $401 \pm 20$ & $520 \pm 25$ & $392 \pm 33$ & $444 \pm 67$ \\
\hline \hline & & & & \\
\hline \hline Ileum & Sham & $\mathrm{R}$ & $\mathrm{R}+\mathrm{ARG}$ & $\mathrm{R}+\mathrm{CIT}$ \\
\hline putrescine & $44 \pm 4$ & $68 \pm 29$ & $128 \pm 23$ & $86 \pm 28$ \\
spermidine & $879 \pm 87$ & $951 \pm 20$ & $1048 \pm 80$ & $909 \pm 131$ \\
spermine & $614 \pm 27$ & $526 \pm 73$ & $479 \pm 29$ & $499 \pm 78$ \\
\hline \hline
\end{tabular}

Four groups of rats were studied: Sham (transected rats fed standard parenteral nutrition), $R$ (resected rats fed standard parenteral nutrition), $R+A R G$ (resected rats fed an arginine-supplemented formula), $R+C I T$ (resected rats fed a citrulline-supplemented formula)

Results are expressed as means \pm SEM. ANOVA + Duncan's test. Values in a row with different superscript letters are statistically different at $\mathrm{p}<0.05$. 


\section{Figure Legends}

Fig 1. Cumulative body weight loss over the duration of total parenteral nutrition (g).

Four groups of rats were studied: Sham (transected rats fed standard parenteral nutrition), R (resected rats fed standard parenteral nutrition), R+ARG (resected rats fed an arginine-supplemented formula), $\mathrm{R}+\mathrm{CIT}$ (resected rats fed a citrulline-supplemented formula).

Results are expressed as means \pm SEM. ANOVA + Duncan’s test

Fig 2. Nitrogen balance over the duration of total parenteral nutrition (mg/72h). Four groups of rats were studied: Sham (transected rats fed standard parenteral nutrition), $R$ (resected rats fed standard parenteral nutrition), $R+A R G$ (resected rats fed an arginine-supplemented formula), $R+C I T$ (resected rats fed a citrulline-supplemented formula). Results are expressed as the difference between daily nitrogen intake and daily urinary nitrogen excretion.

Results are expressed as means \pm SEM. ANOVA + Duncan's test. Values in a row with different superscript letters are statistically different at $\mathrm{p}<0.05$.

\section{Schema: Intestinal-renal axis of arginine metabolism.}

(GLNase, Glutaminase; OAT, ornithine aminotransferase; OCT, ornithine carbamoyltransferase; ARGase, arginase; ARG, arginine; CIT, citrulline; ORN, ornithine; GLN, glutamine; GLU, glutamate; ASS+ASL, argininosuccinate synthetase + lyase). 


\section{Figure 1}

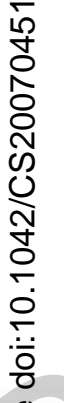

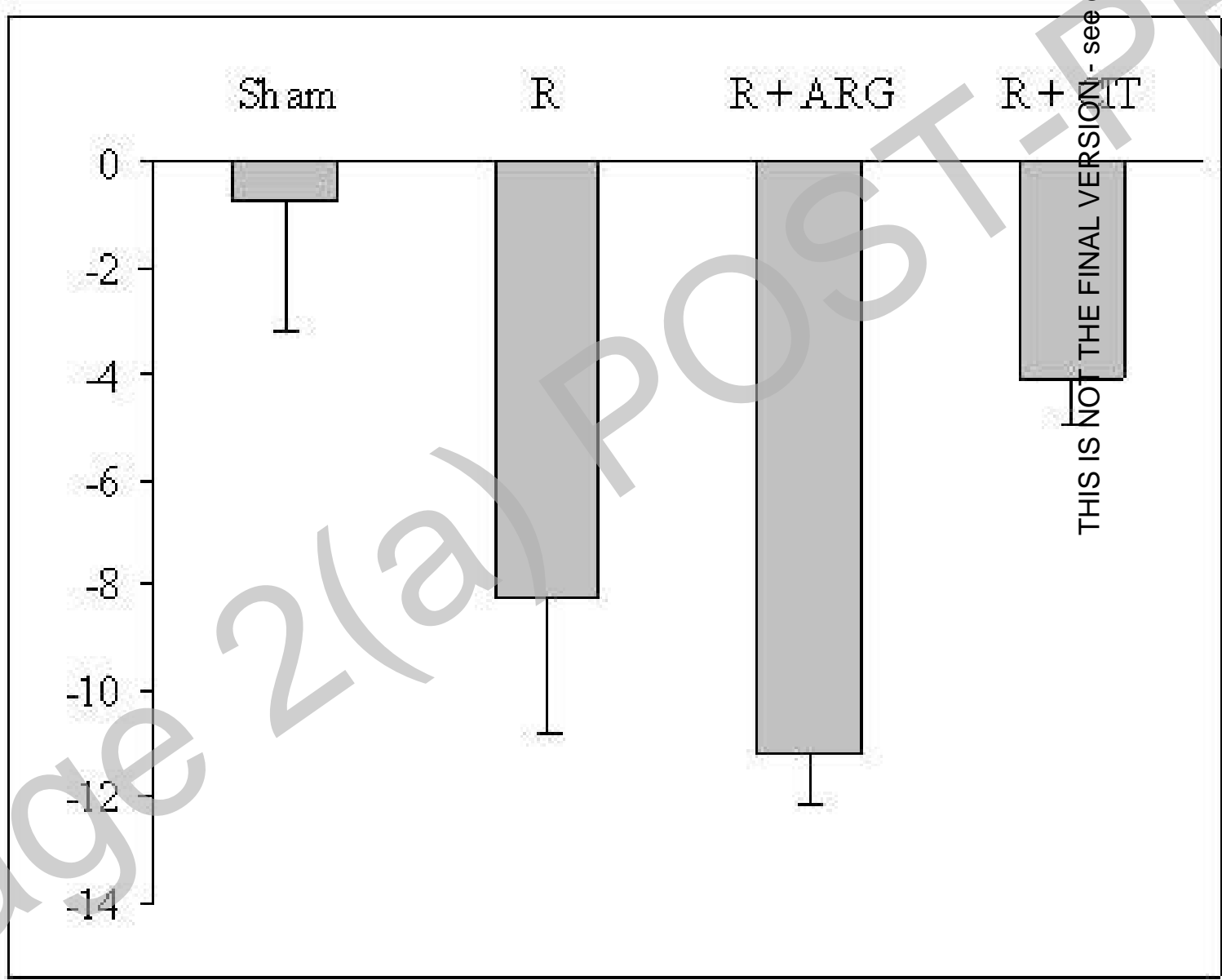




\section{Figure 2}

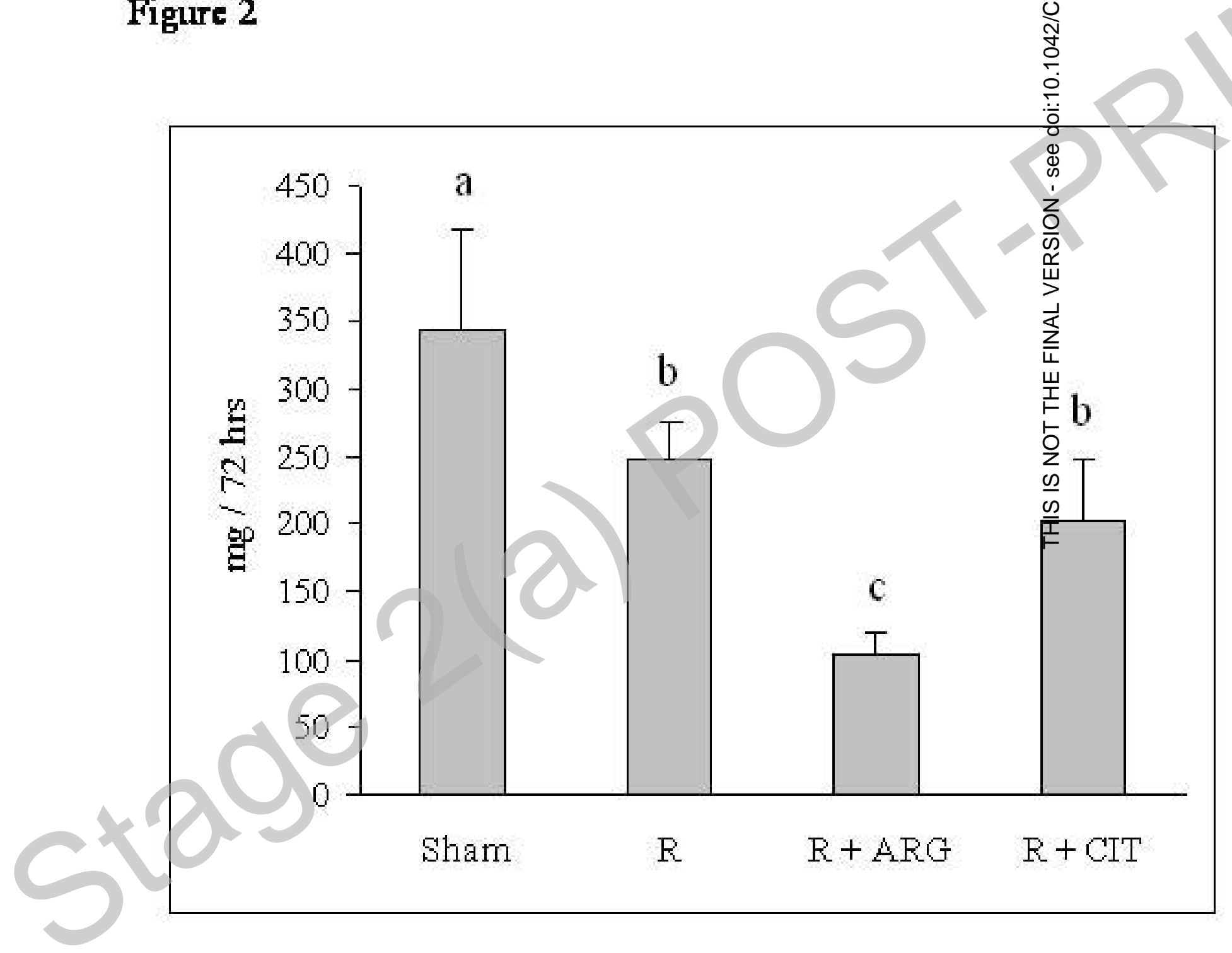




\section{Scheme}

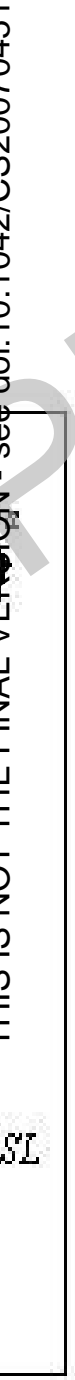

